\title{
BMJ Open A protocol for a randomised controlled trial of the bone response to impact loading or resistance training in young women with lower than average bone mass: the OPTIMA-Ex trial
}

\author{
Conor Lambert, ${ }^{1,2}$ Belinda R Beck, ${ }^{1,2,3}$ Amy T Harding, ${ }^{1,2}$ Steven L Watson, ${ }^{1,2}$ \\ Benjamin K Weeks ${ }^{1,2}$
}

To cite: Lambert C, Beck BR, Harding AT, et al. A protocol for a randomised controlled trial of the bone response to impact loading or resistance training in young women with lower than average bone mass: the OPTIMA-Ex trial. BMJ Open 2017;7:e016983. doi:10.1136/ bmjopen-2017-016983

- Prepublication history for this paper is available online. To view these files please visit the journal online (http://dx.doi. org/10.1136/bmjopen-2017016983).

Received 24 March 2017 Revised 29 May 2017

Accepted 28 June 2017

\section{CrossMark}

${ }^{1}$ Menzies Health Institute Queensland, Griffith University, Gold Coast, Queensland, Australia

${ }^{2}$ School of Allied Health Sciences, Griffith University, Gold Coast, Queensland, Australia

${ }^{3}$ The Bone Clinic, Brisbane, Queensland, Australia

Correspondence to Benjamin K Weeks; b.weeks@griffith.edu.au

\section{ABSTRACT}

Introduction The aim of the 0steoporosis Prevention Through Impact and Muscle-loading Approaches to Exercise trial is to compare the bone response to two known osteogenic stimuli - impact loading exercise and resistance training. Specifically, we will examine the effect of a 10-month, twice-weekly, high-intensity impact loading exercise intervention and a 10-month, twice-weekly, highintensity resistance training intervention on bone mass and strength at clinically important skeletal sites. The intervention groups will be compared against a home-based 'positive' control group. Safety and acceptability of each exercise modality will also be determined.

Methods and analysis Sedentary otherwise healthy young women aged 18-30 years with bone mineral density (BMD) T-scores less than or equal to 0 at the hip and lumbar spine, screened for conditions and medications that influence bone and physical function, will be recruited. Eligible participants are randomised to 10-month, twice-weekly, either supervised high-intensity impact training, high-intensity resistance training or a home-based 'positive' control group. The primary outcome measure will be lumbar spine areal BMD, while secondary outcome measures will include: whole body, femoral neck and regional measures (upper and lower limb) of bone, muscle and fat; anthropometrics; muscle strength and power; quality of life and exercise safety, enjoyment and acceptability. All outcome measures will be conducted at baseline (T0) and 10 months (T10) and will be analysed according to the intention-to-treat principle and per protocol. Ethics and dissemination The study has been granted ethical approval from the Griffith University Human Research Ethics Committee (GU Ref: 2015/775). Standard scientific reporting practices will occur, including publication in peer-reviewed journals. Participant confidentiality will be maintained in all forms of reporting. Trial registration number ACTRN12616001444471.

\section{INTRODUCTION}

Osteoporosis is a progressive metabolic bone disease characterised by the loss of bone mineral density (BMD) and compromised bone strength predisposing to an increased

\section{Strengths and limitations of this study}

- This will be the first trial to investigate the comparative efficacy and safety of high-intensity, progressive impact loading versus high-intensity progressive resistance training programme on determinants of fracture risk of both the upper and lower limbs for healthy young adult women.

- While dual-energy X-ray absorptiometry (DXA)based bone mineral density remains the clinical standard for bone mass estimation, the inclusion of 3D DXA and peripheral quantitative CT outcomes will provide an account of morphological bone adaptations beyond standard bone mineral density measures.

- By necessity, a single investigator will conduct both data collection and participant training which increase the risk of ascertainment bias.

- Given that our cohort comprises healthy young adult women, our findings may not be applicable to men nor to young women suffering from clinical conditions or pathologies.

susceptibility to fracture. ${ }^{1}$ The condition currently affects 1.2 million Australians, ${ }^{2}$ and a further 6.3 million Australians have osteopaenia. ${ }^{2}$ In fact, approximately one in four women aged over 50 and one in two women aged over 80 are osteoporotic. ${ }^{2}$ A recent Burden of Disease analysis concluded that the total costs of osteoporotic fractures over the next 10 years in Australia alone will be $\$ 33.6$ billion. ${ }^{3}$ An important risk factor for osteoporosis in old age is an inadequate accumulation of peak bone mass prior to skeletal maturity. ${ }^{4}$ Epidemiological studies suggest that a $10 \%$ higher peak bone mass will reduce the risk of fracture by $50 \%,{ }^{5}$ thus highlighting the importance of maximising peak bone mass. As peak bone mass occurs by the end of the third decade, ${ }^{6}$ the maximisation of bone 
mass prior to skeletal maturity is an important strategy to prevent osteoporosis in old age.

The skeletal response to exercise is highly dependent on the nature of the activity. Animal research has established that the most osteogenic loads are those that induce high-magnitude bone strains ${ }^{7}$ at high loading rates. ${ }^{89}$ Therefore, it is unsurprising that high-impact exercise (such as landing from a jump) or forceful muscle contractions (such as heavy resistance training) are required to stimulate adaptive changes in human bone. ${ }^{10}$ Some debate has occurred as to the predominant source of the adaptive stimulus-muscle forces or gravity-derived impact loads. ${ }^{11}$ The determination of the most effective source of loading would provide grounds for optimal exercise prescription for bone health. Both resistance training $^{12-14}$ and jump training ${ }^{15-17}$ produce positive effects on bone health in young adult and premenopausal women. BMD targeted exercise interventions in premenopausal women resulted in significant improvements at the femoral neck and lumbar spine with effect sizes of 0.342 and 0.201 , respectively, using a random effects model. ${ }^{18}$ However, there are few direct comparisons of the effectiveness of specific exercise modalities on bone mass in young women. ${ }^{19}$ Furthermore, a comparison of the effect of high-impact loading and heavy resistance training on bone of the upper and lower limbs has not been undertaken.

High-impact weight-bearing exercise, in the form of jumping, has been shown to improve bone mass in young and middle-aged adults. ${ }^{20}{ }^{21}$ Although the effect is often limited to the femoral neck, ${ }^{162223}$ significant improvements in lumbar spine BMD have also been observed. ${ }^{24}$ This is highlighted further by the significant improvement at the femoral neck, but not the lumbar spine based on weighted mean BMD differences of $0.017 \mathrm{~g} /$ $\mathrm{cm}^{2}$ and $-0.001 \mathrm{~g} / \mathrm{cm}^{2}$ from high-impact interventions in premenopausal women. ${ }^{21}$ For premenopausal women, a positive relationship exists between impact intensity and proximal femur BMD change above accelerations of $3.9 \mathrm{~g}$, and greater than $5.4 \mathrm{~g}$ at the lumbar spine. ${ }^{25}$ Such accelerations correspond with the 'high intensity' domain for mechanical loading forces, which is defined as loads greater than four times body weight. ${ }^{26}$ 'High intensity' impact training is therefore likely to be most fruitful for improving bone mass at both clinically relevant skeletal sites.

Although not universally effective, resistance training has also been observed to improve BMD. ${ }^{27-29}$ In contrast with impact exercise, resistance training appears to have a greater effect on the lumbar spine than the hip, exhibiting a weighted mean difference of $0.014 \mathrm{~g} / \mathrm{cm}^{2}$ and $0.001 \mathrm{~g} / \mathrm{cm}^{2}$, respectively. ${ }^{30}$ Where studies have shown no effect or only maintenance of $\mathrm{BMD}$, resistance training intensity was low to moderate, that is, $60 \%$ or less of 1 repetition maximum (RM).$^{1231}$ In fact, the American College of Sports Medicine position stand on physical activity and bone health and the Exercise and Sports Science Australia position stand on exercise for osteoporosis both state that resistance training must be site specific and of high intensity to cause an increase in bone mass in young adult and premenopausal women. ${ }^{32}{ }^{33}$ Nevertheless, most studies have examined resistance training at moderate intensities, such that high-intensity progressive resistance training ( $>85 \%$ 1 RM, 3-5 reps) has not been fully explored for bone in this demographic.

Loads from the squat and deadlift exercises at the hip and spine can reach 5-8 times body weight, ${ }^{34} 35$ suggesting adequate force to increase bone mass. Two recent studies specifically examined the effect of high-intensity resistance training on bone mass of young adult women. One focused on machine hack squats with a regime of four sets of 3-5 reps at $>85 \%$ $1 \mathrm{RM}$ and produced significant within-group change in lumbar spine and total hip BMD, but no betweengroup difference at follow-up. ${ }^{36}$ The second study used large multijoint exercises such as bench press, squats and deadlifts at $67 \%-95 \% 1 \mathrm{RM}$ over a 24 -week training period in college-aged men and women; however, unremarkable findings were reported for the female participants. ${ }^{37}$ The short duration and modest sample sizes of both studies, however, likely limit the strength of their findings. Adequately powered randomised controlled trials (RCTs) employing sufficiently long-duration resistance training of optimum type, intensity and volume of loading are required. ${ }^{18} 3038$

The most common sites of osteoporotic fracture are the spine, hip and wrist. ${ }^{39}$ Spine and hip fractures account for the greatest morbidity and mortality but occur most often in older people, while wrist fractures occur earlier in life and carry a lifetime risk of $15 \%$ in women. ${ }^{40}$ In spite of the evident burden of wrist fractures, the upper limb has been largely overlooked as a target for bone health interventions. Moreover, few studies have looked specifically at the upper limb bone response to resistance training, ${ }^{4142}$ or the effect of impact loading on upper limb bones. ${ }^{43}$ Observational data demonstrate that participation in weight lifting exercise is positively associated with bone density of both the upper and lower limbs. ${ }^{44}{ }^{45}$ Furthermore, those who participate in fight sports have higher bone mineral density than controls, including greater arm bone mineral density than controls and active/sporting populations. ${ }^{46-49}$ As the efficacy of punching-based upper limb impact exercises on bone health has not been examined in a randomised controlled study design, causality is yet to be confirmed.

The proposed project takes a novel approach to comparing the bone response to high-intensity impact loading exercise and high-intensity resistance training in a human exercise model to clarify relative effects. The determination of a most effective source of loading would provide grounds for optimal prescription for osteogenic exercise strategies for young adult women. 


\section{METHODS AND ANALYSES}

\section{Study aims}

The aim of the Osteoporosis Prevention Through Impact and Muscle-loading Approaches to Exercise (OPTIMA-Ex) trial is to examine and compare the efficacy of a 10-month training intervention of two known osteogenic loading methods, predominantly impact loading versus predominantly muscle loading exercise in young women with lower than average bone mass. Additionally, we will determine the safety and acceptability of each exercise training mode. Intervention group responses will be compared with each other, and against a homebased 'positive' control group. For our primary outcome measure of lumbar spine BMD, we hypothesise that the resistance training group will show greater improvements when compared with the impact training group and that both intervention groups will show greater improvement than the home-based 'positive' control group. For secondary outcome measures, we hypothesise greater improvement of hip BMD in the impact group compared with the resistance training group; and for upper and lower limb peripheral bone strength measures, there will be no difference between the intervention groups, but the improvement will be greater in these groups compared with the home-based 'positive' control group. Furthermore, we hypothesise that there will be no difference between groups for quality of life, adverse events, safety, compliance, acceptability and enjoyment.

\section{Study design}

The OPTIMA-Ex trial will be conducted as a three-arm, single-blinded, single-centre randomised controlled exercise intervention trial. Participant flow is illustrated in figure 1. Eligible participants will be randomised to 10-month, twice-weekly, supervised high-intensity impact training, supervised high-intensity resistance training or a home-based 'positive' control group. A 10-month training period has been adopted to provide sufficient time for changes to be detected using densitometry, as a bone remodelling cycle takes approximately 4-6 months with additional time required to mineralise new osteoid. ${ }^{50}$ It has been suggested that a minimum of $6-8$ months is required to achieve a new steady-state bone mass that is measurable with densitometry. ${ }^{32}$ The study protocol, where applicable, adheres to the Standard Protocol Items: Recommendations for Interventional Trials guidelines for clinical trial protocols. ${ }^{51}$ Outcome measures will be conducted at baseline (T0) and 10 months (T10).

\section{Setting and recruitment}

Data collection and training will occur in the School of Allied Health Sciences, Griffith University, Gold Coast campus, Queensland, Australia. Baseline and follow-up assessments will take place in the Bone Densitometry Research Laboratory. The supervised impact training sessions will take place in the Movement Laboratory Research Facility and the supervised resistance training sessions will be conducted in the Strength Training
Research Facility. Recruitment will be achieved with the use of local print media and radio, social media, flyers, study website (www.optima-ex.com) and word-of-mouth. Formal recruitment for the study began in November 2016 and will continue until November 2017.

\section{Participant eligibility and screening}

Sedentary, otherwise healthy, young adult women (18-30 years) with below average bone mass (BMD T-score less than or equal to 0 at the lumbar spine and hip) will be recruited for the OPTIMA-Ex trial. Participants must be willing to undertake a 10-month exercise programme and be randomly allocated to any of the three training groups. Participants will be unaware of the specific training exercises implemented in the groups other than their own. Furthermore, all participants will be unaware of the hypothesised effects of each group. Volunteers will be excluded based on the following criteria: musculoskeletal or medical conditions affecting the ability to participate in high-intensity physical activity, medications (other than contraceptive medications) or medical conditions known to effect bone health, change of contraceptive medication in the past 12 months, cancer, uncontrolled cardiovascular disease, greater than two X-rays in the past 12 months and current regular participation in an exercise programme known to influence bone or previous competitive or regular participation (more than once per week for 1 year or one full season) in resistance training or boxing (including other fight sports and martial arts), gymnastics/dance sports (gymnastics, ballet, aerobics, cheerleading), court sports (volleyball, basketball, netball), ball sports (Australian rules, rugby league/ union, soccer, touch) or racquet sports (badminton, squash, tennis) over the past 4 years.

Initial screening will be conducted by the investigator via phone to establish eligibility based on the exclusion criteria. Disclosure of pre-existing medical conditions or injuries that may increase the risk of an adverse event occurring during vigorous intensity exercise (ie, heavy resistance training or high-impact activity) will result in exclusion from the study. Any current medications will be carefully examined for any known effect on bone metabolism. Once deemed eligible from the initial screening, potential participants will be asked to attend baseline testing at the Bone Densitometry Research Laboratory at Griffith University. Prior to testing, each participant will provide written informed consent. Participants will be eligible for inclusion if they exhibit below average bone mass (BMD T-score less than or equal to 0 at the lumbar spine and hip). Participants will be withdrawn from the trial if: (1) consent is withdrawn, (2) change in medication of relevance to bone (ie, contraceptive medication) occurs, (3) injury or illness occurs preventing further participation, (4) advised to cease training by a medical professional (ie, their general practitioner) or (5) additional exercise deemed as frequent (ie, weekly), osteogenic in nature or moderate to vigorous in intensity is commenced during the trial. 


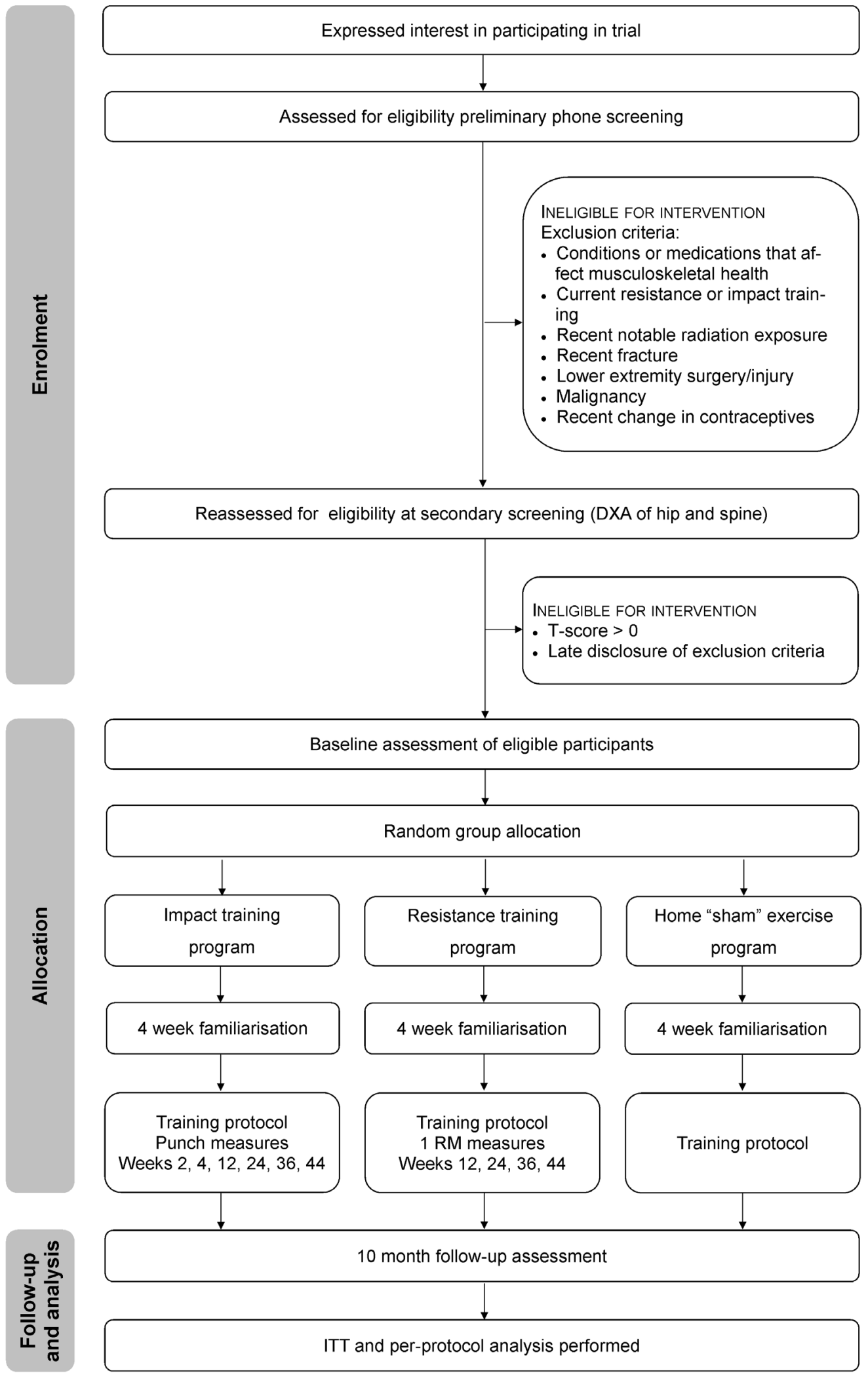

Figure 1 Proposed participant flow (CONSORT diagram). DXA, dual-energy X-ray absorptiometry; ITT, intention-to-treat; 1 $\mathrm{RM}$; one repetition maximum. 


\section{Randomisation, allocation and blinding}

Eligible participants will be randomised to either high-intensity impact training, high-intensity resistance training or home-based 'positive' control exercise. A random number generator (Microsoft Excel, Microsoft, Redmond, Washington, USA) will be used to generate either a 0,1 or 2 to correspond with home-based 'positive' control group, high-intensity resistance training group and the high-intensity impact training group, respectively. The randomisation allocation for each participant will be conducted in advance by an individual independent of the study team. The allocation sequence will be sealed in opaque envelopes and locked in a filing cabinet remote from the testing site. The independent individual will provide envelopes sequentially at the end of the baseline testing session and remain present to witness group allocation. Therefore, the investigator performing baseline assessments will be blinded to the allocation sequence, which will only be revealed after completion of baseline evaluations. Participants in the three exercise groups will perform training separately, and will not be aware which exercise programme is hypothesised to be more effective. Follow-up testing will not be assessor blinded to maintain the highest level of test-retest reliability.

\section{Exercise interventions}

High-intensity progressive impact training programme

Participants allocated to the impact training intervention group will attend twice-weekly, instructor-led, high-intensity impact training at our exercise facility. Each session will be approximately $40 \mathrm{~min}$ in duration and performed on non-sequential days. A maximum trainer-to-participant ratio of $1: 8$ will be maintained.

To ensure a safe transition, the first month of training will serve as familiarisation to impact activity. Low-load jumping exercise with a focus on controlled jumping and landing with safe movement patterns will be taught with the view to progress to more advanced jumping techniques through the programme. Participants will also be taught correct punching technique and various punching combinations using boxing gloves (Pro Bag Busters, Punch Equipment, Australia) and focus pads (Thumpas Focus Pads, Punch Equipment, Australia) with the view to progress to more advanced punching combinations on a 5-foot heavy bag (Trophy Getters Boxing bag, Punch Equipment, Australia) with padded hand wraps (Punchfit Quickwraps, Punch Equipment, Australia) alone in both orthodox and southpaw position. Such familiarisation is necessary to reduce the likelihood of injuries. From months 2-10, participants will be able to comfortably perform the fundamental upper limb (jab, cross and hook) and lower limb (jump, hop, drop jump) impact exercises. High-intensity impact has been previously defined as ground reaction forces of greater than four times the body weight ${ }^{26}$ and, therefore, the lower limb loading intervention includes impact exercises that produce impacts of greater than four times the body weight based on previously published data from our laboratory. ${ }^{52}$ The fundamental exercises will be performed throughout the remainder of the intervention period with progressively increasing height of drop jumps from $15 \mathrm{~cm}$ to $80 \mathrm{~cm}$ over the duration of the trial with increases every 7 weeks. The complexity of jumps and hops will occur with increases in hurdle height, changes in directionality of task as well as force of landing with participants progressing from shod to barefoot after the familiarisation phase (week 4). Furthermore, the power and complexity of punching combinations will increase every 7 weeks. Measures of punch force and velocity will take place at 2, 4, 12, 24, 36 and 44 weeks using a GymAware device (Kinetic Performance, Canberra, Australia) to ensure that participants continue to increase the load and speed of their maximal punches throughout the training intervention. Measures of vertical ground reaction force for all jumps included in the intervention will occur at the completion of the intervention using a floormounted force plate (Advanced Mechanical Technology, Watertown, Massachusetts, USA), capturing at $1000 \mathrm{~Hz}$ using Vicon Nexus software V.1.8 (Vicon, Oxford Metrics, Oxford, UK). Training diaries will be used to record attendance, exercise compliance, muscle soreness, adverse events and any changes to diet, exercise or medications.

\section{High-intensity progressive resistance training programme}

Participants allocated to the resistance training intervention group will attend twice-weekly, instructor-led, high-intensity resistance training at our exercise facility. Each session will be approximately $45 \mathrm{~min}$ in duration and performed on non-consecutive days. A maximum trainer-to-participant ratio of 1:8 is maintained.

To ensure a safe transition into the programme for those unaccustomed to exercise training, the first month of the intervention will serve as familiarisation. Low-load exercise variants with a focus on controlled movement will be undertaken to ensure that participants can demonstrate safe lifting technique. A graduated transition serves to ensure proper form so that technique errors known to increase injury risk are avoided. From months 2-10, participants will be able to comfortably perform the six fundamental compound exercises (deadlift, back squat, bench press, overhead press, bent over row and calf raise) using Olympic weights. The six fundamental exercises will be performed throughout the duration of the intervention period with a focus on progressively increasing weight to maintain a minimum of $85 \% 1 \mathrm{RM}$ for five sets of 3-5 repetitions. If a weight is able to be lifted for five sets of five repetitions, the load will be increased by $2.5 \mathrm{~kg}$ for at least one set in the following session. Measurements of $1 \mathrm{RM}$ will take place every 12 weeks to ensure sufficient progression of resistance training exercises. During months 2 and 3 (prior to the first 1 RM testing), participants will perform each exercise at five sets of $3-5$ repetitions as previously described, lifting the maximum weight possible for five repetitions while maintaining correct form. Training diaries will be used to record attendance, exercise compliance, weights lifted, muscle soreness, adverse events and any changes to diet, exercise or medications. 


\section{Home-based 'positive' control exercise programme}

The home-based exercise programme consists of a low-load and low-intensity circuit training regime to serve as a positive control. Participants will be asked to exercise twice per week for approximately $30-45 \mathrm{~min}$. The sessions consist of shadow boxing and low-load body weight exercises (abdominal crunches, bridges, side planks, front planks as well as upper and lower limb stretches) in a low-intensity circuit training format. Participants will warm up with a 10-min walk, followed by a low-load and low-intensity circuit, building from one circuit per session to three circuits per session by the end of 10 months with 1-2 min rest in between each circuit. Each session will be followed by gentle, targeted stretches as a cool down. As is the case for the supervised groups, the first month of the programme will serve as familiarisation to ensure a safe transition to the activity. Participants will be instructed on how to perform the exercises at their initial testing session and will be provided with a training diary and a handout with photographs of all exercises. Participants also have access to the OPTIMA-Ex YouTube channel, which will step them through each shadow boxing exercise progression so they can follow along at their own pace. The YouTube channel is unlisted and can only be accessed via the URL which will be sent to the home-based group via email. Training diaries will be used to record exercise compliance, muscle soreness, adverse events and any changes to diet, exercise or medications.

Weekly emails will be sent to participants to keep regular contact, to monitor progress and to reinforce reporting of training diary entries.

\section{Outcome measures}

A single investigator (CL) will conduct all outcome measures at baseline and 10-month follow-up for all participants using identical equipment and standardised procedures (table 1).

\section{Primary outcome}

The primary outcome will be the change in lumbar spine areal bone mineral density (aBMD; Dual-energy X-ray Absorptiometry (DXA), Medix DR, Medilink, France).

\section{Secondary outcomes}

Secondary outcomes will include: changes in bone strength indices of whole body, proximal femur, lumbar spine, radius, tibia and calcaneus; the comparison between upper and lower extremity bone strength outcomes; changes in anthropometrics and body composition; changes in muscle strength and power; as well as changes in quality of life, exercise enjoyment and acceptability.

\section{Bone strength indices}

DXA (Medix DR, Medilink, Perols, France) will be used to determine aBMD, bone mineral content and bone area for whole body, lumbar spine, bilateral proximal femur and bilateral distal forearm following standard procedures. For the whole body scan, participants will be supine with their arms by their sides, and legs extended. The lumbar spine scan requires participants to be supine, with their hips flexed approximately $60-70$ degrees using a foam block under their legs to ensure the lumbar spine curvature is flattened. For the proximal femur scans, participants will be supine with feet separated and the test hip internally rotated to 20 degrees using a positioning device. This process will be repeated for the contralateral limb, as both proximal femora will be examined. The forearm scans will require the participant to be seated next to the DXA scanner, with their shoulder abducted and elbow flexed so that the pronated forearm rests on the scanning bed. This process will be repeated for the contralateral limb, as both forearms will be examined. All scans will be acquired through an automated procedure initiated by the investigator from a designated computer console. The coefficients of variation for DXA-derived aBMD of whole body, lumbar spine, total hip (left and right), femoral neck (left and right), distal forearm (left and right) range from $0.83 \%$ to $2.21 \%$. Data analysis for DXA scans will be undertaken with host software (Medix DR, Medilink, Eaxiz software V.4.0.2.4). Geometric and volumetric density parameters of the proximal femur will be calculated using 3D hip analysis software (DMS Group, Mauguio, France) from the standard hip scans.

Indices of bone strength, including geometric parameters and volumetric bone mineral density, will be determined from bilateral radius and tibial scans using peripheral quantitative CT (pQCT, XCT-3000, Stratec Medizintechnik GmbH, Pforzheim, Germany). For both radius and tibial scans, the limb will be positioned in the gantry on a Perspex support (with bubble wrap to separate limb from Perspex) and secured with Velcro straps to reduce the likelihood of movement. To identify scanning regions, ulna and tibial length will be measured prior to positioning and a scout scan will be used to locate the distal end plates of the radius and tibia, respectively. Data analysis for pQCT scans will be undertaken with host software (Stratec XCT-3000, Medizintechnick GmbH) and loop analysis techniques defined by Bone Diagnostic (Spring Branch, Texas, USA). For radius scans, the $4 \%$ and $66 \%$ sites will be used, whereas for tibial scans, the $4 \%$ and $38 \%$ sites will be examined. These regions have been selected in order to assess trabecular and cortical bone as well as muscle, and to be comparable with relevant recent pQCT studies using the XCT-3000 (Stratec, Medizintechnik GmbH) ${ }^{53-56}$ The coefficients of variation for the pQCT bone outcomes range from $0.74 \%$ to $2.71 \%$ at the $4 \%$ tibial site; from $0.21 \%$ to $1.40 \%$ at the $38 \%$ tibial site; from $0.96 \%$ to $5.05 \%$ at the $4 \%$ radius site; and from $0.62 \%$ to $3.27 \%$ at the $66 \%$ radius site.

Parameters of calcaneal bone quality will be assessed using Quantitative Ultrasonometry (Lunar Achilles InSight, GE Healthcare, Wisconsin, USA). Participants will be seated in a chair, with the knee of the limb to be examined flexed at approximately 60 degrees, the hip neither abducted nor adducted and the heel positioned midway between the walls of the heel well. Participant details will be entered and a scout image will be obtained to ensure correct placement of the participant's heel 
Table 1 Summary of outcome measures to be collected

\begin{tabular}{|c|c|c|}
\hline Measure & Unit & Data collection method \\
\hline \multicolumn{3}{|l|}{ Primary outcome measure } \\
\hline Lumbar spine aBMD & $\mathrm{g} / \mathrm{cm}^{2}$ & DXA (Medix DR, Medilink, Perols, France) \\
\hline \multicolumn{3}{|l|}{ Secondary outcome measures } \\
\hline \multicolumn{3}{|l|}{ Bone strength indices } \\
\hline Whole body (aBMD; BMC; area) & $\mathrm{g} / \mathrm{cm}^{2} ; \mathrm{g} ; \mathrm{cm}^{2}$ & DXA (Medix DR, Medilink) \\
\hline Lumbar spine (BMC; area) & $\mathrm{g} ; \mathrm{cm}^{2}$ & \\
\hline $\begin{array}{l}\text { Proximal femur-femoral neck, trochanter and total hip } \\
\text { regions (aBMD; BMC; area) }\end{array}$ & $\mathrm{g} / \mathrm{cm}^{2} ; \mathrm{g} ; \mathrm{cm}^{2}$ & \\
\hline Distal forearm (aBMD, BMC) & $\mathrm{g} / \mathrm{cm}^{2} ; \mathrm{g}$ & \\
\hline Femoral neck (vBMD; cortical thickness; volume) & $\mathrm{g} / \mathrm{cm}^{3} ; \mathrm{mm} ; \mathrm{cm}^{3}$ & $\begin{array}{l}\text { 3D hip software (DMS Group, Mauguio, } \\
\text { France) }\end{array}$ \\
\hline Total hip (vBMD; cortical thickness; volume) & $\mathrm{g} / \mathrm{cm}^{3} ; \mathrm{mm} ; \mathrm{cm}^{3}$ & \\
\hline $\begin{array}{l}\text { Forearm-4\%,66\% (content; vBMD; CSA; thickness; } \\
\text { circumference; polar section modulus; polar SSI; BSI) }\end{array}$ & $\begin{array}{l}\mathrm{mg} ; \mathrm{mg} / \mathrm{cm}^{3} ; \mathrm{mm}^{2} ; \mathrm{mm} \\
\mathrm{mm} ; \mathrm{mm}^{2} ; \mathrm{mm}^{3} ; \mathrm{g}^{2} / \mathrm{cm}^{4}\end{array}$ & $\begin{array}{l}\text { pQCT (XCT-3000, Stratec Medizintechnik } \\
\text { GmbH, Pforzheim, Germany) }\end{array}$ \\
\hline $\begin{array}{l}\text { Leg }-4 \% \text { and } 38 \% \text {, (content; vBMD; CSA; thickness; } \\
\text { circumference; polar section modulus; polar SSI; BSI) }\end{array}$ & $\begin{array}{l}\mathrm{mg} ; \mathrm{mg} / \mathrm{cm}^{3} ; \mathrm{mm}^{2} ; \mathrm{mm} ; \\
\mathrm{mm} ; \mathrm{mm}^{2} ; \mathrm{mm}^{3} ; \mathrm{g}^{2} / \mathrm{cm}^{4}\end{array}$ & \\
\hline Calcaneal (BUA; SOS; SI) & $\mathrm{dB} / \mathrm{MHz} ; \mathrm{m} / \mathrm{s}$; unitless & $\begin{array}{l}\text { QUS (Lunar Achilles InSight, GE } \\
\text { Healthcare, Wisconsin, USA) }\end{array}$ \\
\hline \multicolumn{3}{|l|}{ Anthropometrics } \\
\hline Height & $\mathrm{m}$ & $\begin{array}{l}\text { Wall mounted stadiometer (Seca, } \\
\text { Hamburg, Germany) }\end{array}$ \\
\hline Weight & $\mathrm{kg}$ & Mechanical beam scale (Seca) \\
\hline Waist circumference & $\mathrm{mm}$ & $\begin{array}{l}\text { Steel tape (Lufkin Executive Thinline, } \\
\text { Apex, USA) }\end{array}$ \\
\hline
\end{tabular}

Body composition

$\begin{array}{lll}\text { Whole body (lean mass; fat mass; body fat percentage) } & \mathrm{g} ; \mathrm{g} ; \% & \text { DXA (Medix DR, Medilink) } \\ \text { Forearm-66\% (muscle CSA; muscle density) } & \mathrm{mm}^{2} ; \mathrm{mg} / \mathrm{cm}^{3} & \text { pQCT (XCT-3000, Stratec Medizintechnik } \\ & & \text { GmbH) }\end{array}$

Leg-66\% (muscle CSA; muscle density) $\quad \mathrm{mm}^{2} ; \mathrm{mg} / \mathrm{cm}^{3}$

Muscle strength

\begin{tabular}{|c|c|c|}
\hline Lower limb isometric strength & $\mathrm{kg}$ & $\begin{array}{l}\text { Leg dynamometer (TTM Muscle Metre, } \\
\text { Tokyo, Japan) }\end{array}$ \\
\hline Back extensor isometric strength & $\mathrm{kg}$ & $\begin{array}{l}\text { Dynamometer (Lafayette Manual Muscle } \\
\text { Testing Systems, USA) }\end{array}$ \\
\hline Hand grip isometric strength & $\mathrm{kg}$ & $\begin{array}{l}\text { Dynamometer (JAMAR Plus +, Patterson } \\
\text { Medical, Sammons Preston, Bolingbrook } \\
\text { Illinois, USA) }\end{array}$ \\
\hline
\end{tabular}

Muscle Power

$\begin{array}{ll}\text { Countermovement vertical jump } & \mathrm{N} \cdot \mathrm{s} / \mathrm{kg}^{2} \\ & \text { Load cell (Advanced Mechanical } \\ & \text { Technology, Watertown, Massachusetts, } \\ & \text { USA) }\end{array}$

Physical activity

\begin{tabular}{lll}
$\begin{array}{l}\text { Bone-specific physical activity (current, past and } \\
\text { lifetime) }\end{array}$ & unitless & BPAQ \\
Calcium Consumption & mg/day & AusCal questionnaire \\
$\begin{array}{l}\text { Daily calcium intake } \\
\text { Quality of Life }\end{array}$ & AQoL-6D questionnaire \\
$\begin{array}{l}\text { Health-related quality of life } \\
\text { Exercise acceptability }\end{array}$ & See text & \\
\hline
\end{tabular}


Table 1 Continued

\begin{tabular}{lll}
\hline Measure & Unit & Data collection method \\
\hline Exercise enjoyment, & Overall score & PACES questionnaire \\
Barriers and facilitators & See text & Semistructured interviews \\
Safety and compliance & & \\
Safety & See text & Training diary \\
Compliance & $\%$ & \\
\hline
\end{tabular}

aBMD, areal bone mineral density; AQoL, Assessment of Quality of Life; BMC, bone mineral content; BPAQ, Bone-specific Physical Activity Questionnaire; BSI, bone strength index; BUA, broadband ultrasound attenuation; CSA, cross-sectional area; DXA, dual-energy X-Ray absorptiometry; PACES, Physical Activity Enjoyment Scale; PQCT, peripheral quantitative CT; QUS, quantitative ultrasound; SI, stiffness index; SOS, speed of sound; $\mathrm{VBMD}$, volumetric bone mineral density.

before the full examination is undertaken via a device-automated procedure. The coefficients of variation for the quantitative ultrasound measures of the calcaneus range from $0.30 \%$ to $2.44 \%$.

\section{Anthropometrics and body composition}

Height and weight will be determined using a wallmounted stadiometer (Seca, Hamburg, Germany) and mechanical beam scale (Seca), respectively. National Institute of Health guidelines ${ }^{57}$ will be followed for the measurements of waist circumference. Whole body scans from DXA (Medix DR, Medilink) will be used to determine parameters of body composition, including body fat percentage, lean mass and fat mass. Upper and lower extremity pQCT scans (Stratec XCT 3000, Medizintechnick $\mathrm{GmbH}$ ) will be used to examine muscle cross-sectional area and muscle density of the forearm and leg at the $66 \%$ site.

\section{Muscle strength}

Lower extremity maximal isometric strength will be assessed with a leg strength dynamometer (TTM Muscle Metre, Tokyo, Japan). Participants will stand on a platform with their knees flexed to $115^{\circ}$ and their back against the wall. They will then be instructed to grasp a bar connected by a chain to the platform with arms fully extended, with their back and head against the wall and attempt to stand upright by pushing only with their legs. The test has been shown to have excellent test-retest reliability (Pearson's $r=0.97, \mathrm{p}<0.001$ ) and has been validated against the current gold standard of isometric muscle strength, isokinetic dynamometry (Pearson's $r=0.84$, $\mathrm{p}<0.001$ ) (unpublished data, conference abstract). ${ }^{58}$

A handheld dynamometer (Lafayette Manual Muscle Testing Systems, Lafayette, IN, USA) will be used to measure maximal isometric back extensor strength. The participant will stand with their back to the wall midway between two designated anchor points. An inelastic strap will then be firmly secured across the pelvis approximately $1 \mathrm{~cm}$ below the anterior superior iliac spine to prevent movement away from the wall during the trial. To perform the task, the participant will fold their arms across their chest, flexing forward to allow placement of the hand held dynamometer between the seventh thoracic vertebra and the wall. The participant will then push back as hard as possible for $3 \mathrm{~s}$. This assessment of back extensor strength demonstrated excellent long-term test-retest reliability (Pearson's $r=0.926$ ) and has been validated against isokinetic dynamometry (Pearson's $r=0.853) .^{59}$

Maximal grip strength will be assessed using an isometric handheld dynamometer (JAMAR Plus+, Patterson Medical, Sammons Preston, Bolingbrook, Illinois, USA). Participants will sit upright in a chair with feet touching the floor, knees and hips at $90^{\circ}$, their arm by their side and neutrally rotated, elbow flexed to $90^{\circ}$, with the forearm and wrist in a neutral position. Participants will then squeeze the handheld dynamometer with maximum effort.

\section{Muscle power}

A counter movement vertical jump test will be used to examine lower extremity muscle power. Participants will be required to complete a 5-min warm-up on a cycle ergometer (Ergomedic 818E, Monark, Sweden) at 50 watts, prior to the maximal vertical jump test. Participants will then perform a maximal vertical jump on a floor-mounted force plate (Advanced Mechanical Technology). Three-dimensional ground reaction forces will be captured at $1000 \mathrm{~Hz}$ using Vicon Nexus software V.1.8 (Vicon, Oxford Metrics). Using previously described methods, ${ }^{60}$ vertical ground reaction force from the point of stationary standing (prejump) to the point of take-off will be used to calculate impulse.

\section{Bone-specific physical activity}

The Bone-specific Physical Activity Questionnaire $(\mathrm{BPAQ})^{61}$ is a self-report questionnaire to quantify current and historical physical activity according to its osteogenic effect. Participants will be asked to record all activities they have participated in for a year or more throughout their lifetime, as well as list all activities they have engaged in regularly over the past 12 months. The questionnaire is scored using a custom-designed Microsoft Visual Basic executable programme to generate a bone-specific score from algorithms that rank and weight activities based on rates and magnitudes of loading (http://www. fithdysign.com/BPAQ/). A high level of reliability has 
been demonstrated with this instrument, with intraclass correlation coefficients of $0.92-0.97 .^{62}$

\section{Dietary calcium intake}

Daily calcium intake, including supplementation, will be estimated with the AusCal questionnaire. The AusCal is a calcium-focused diet questionnaire specific to the Australian $\operatorname{diet}^{63}$ that employs a list of calcium-rich food items to record consumption over the past 12 months, including frequency (per day, week or month) and approximate serving size. Questionnaires are scored using a customwritten FoodWorks software (V.7, Xyris Software, Brisbane, Australia) to estimate average daily calcium intake (mg per day).

\section{Quality of life}

The Assessment of Quality of Life questionnaire (AQoL) will be used to measure participant's health-related quality of life. The instrument has six separately scored dimensions, with variable item numbers and response levels to produce a simple global 'utility' score. ${ }^{64}$ The AQoL has high internal consistency $(\alpha=0.81) .{ }^{65}$ Unlike other generic utility instruments, a unique feature of the AQoL is that the utility weights have been derived from an Australian population sample ${ }^{66}$ and norms of the Australian population are available. ${ }^{67}$ As a utility measure, it has been established that the minimal clinically important difference of the AQoL is $0.06 .{ }^{67}$

\section{Exercise enjoyment and acceptability}

Participants' perceptions of physical activity participation and enjoyment will be recorded using the modified Physical Activity Enjoyment Scale (PACES) developed by Mullen and colleagues. ${ }^{68}$ The PACES is an eight-item self-report questionnaire which requires participants to select the corresponding number which matches their feelings towards physical activity on a seven-point Likert scale, with higher numbers relating to perceived enjoyment. Furthermore, semistructured interviews will be used to assist in the determination of exercise enjoyment, acceptability and identify potential barriers and facilitators to exercise participation in all three groups. The interviews will be led by an independent investigator immediately prior to follow-up testing so that individual study results do not influence the participant's responses. Dialogue will be recorded digitally with participant consent and subsequently transcribed. Transcripts from each interview will be coded for emergent themes (NVivo, V.10, QRS International Pty Ltd) to determine acceptability as well as any barriers and facilitators to participation in the exercise programme.

\section{Safety and compliance}

All participants will have a personal training diary containing a 10-point visual analogue scale to rate their degree of muscle soreness prior to each training session and space to note any changes to diet, health, physical activity or medications from the previous training session. All injuries other than muscle soreness (including those that occur outside of the training programme) will be recorded. Programme compliance will be examined through attendance logs where $100 \%$ compliance is defined as completion of 88 sessions over 44 weeks or 10 calendar months. Any adverse events or injuries sustained during sessions will be documented by the supervising physiotherapist and monitored for the duration of the intervention. If further management is required, the study investigator will refer an injured participant to the University Physiotherapy and Active Health Centre.

\section{Ethics and dissemination}

Ethical approval was given by the Griffith University Human Research Ethics Committee (GUHREC) (GU Ref: 2015/775) and the study has been registered with the Australian New Zealand Clinical Trials Registry (Trial number ACTRN12616001444471). Compulsory annual progress reports will be submitted to the GUHREC as part of the national research ethics framework. A data monitoring committee was not deemed necessary as the proposed study is not a pharmaceutical trial and safety oversight is provided directly by the GUHREC. Adverse events will immediately be reported directly to the GUHREC, in accordance with the University Code for the Responsible Conduct of Research, and the Australian Code for the Responsible Conduct of Research developed by the National Health and Medical Research Council.

Standard scientific reporting practices will occur, including presentations at discipline-specific conferences and publication in peer-reviewed journals. At the end of the trial, each participant will be provided with a summary of study findings including a breakdown of their own individual results. There are no planned interim analyses for the OPTIMA-Ex trial. Participant confidentiality will be maintained in all forms of reporting.

\section{Data integrity}

All participant data will be deidentified for analysis and all publications arising from OPTIMA-Ex trial to ensure participant confidentiality. All participants will be assigned a unique identification sequence for the trial. Hard copy records will be stored in a secure, swipe-card restricted access laboratory (Bone Densitometry Research Laboratory), while electronic data will be securely stored on password protected computers at Griffith University. No contractual agreements exist to limit data set access; deidentified data will be shared on a case-by-case basis. The management, storage and retention of research data will comply with the Griffith University Code for the Responsible Conduct of Research.

\section{Data analyses and statistical considerations}

SPSS V.24.0 (SPSS, Chicago, Illinois, USA) will be used for all statistical procedures. Descriptive statistics will be generated for participant characteristics, biometrics and all dependent measures. Between-group comparisons will be made using repeated measures analysis of covariance where age, height, weight, baseline values, contraceptive 
medication use, calcium intake and compliance serve as covariates. $\chi^{2}$ analyses will be employed for non-parametric data. All participants who have been randomised will be included in the final analysis at follow-up, irrespective of compliance or withdrawal from the programme, in accordance with the principles of an intention-to-treat analysis. Full information maximum likelihood estimation will be employed for missing data. Per-protocol comparisons will be made for all outcome measures between the high-intensity impact training and the high-intensity resistance training groups to examine treatment efficacy. Statistical significance will be indicated by $p \leq 0.05$. Sample size for the OPTIMA-Ex trial is based on the least significant change (LSC) that is detectable from our device (Medix DR, Medilink) for our primary outcome measure of lumbar spine aBMD. The LSC for BMD that can be detected with $95 \%$ confidence is calculated as $2.77 \times$ coefficient of variance $(\mathrm{CV})$ as recommended by the International Society for Clinical Densitometry. ${ }^{69}$ Therefore, based on the CV of our device for female lumbar spine aBMD $(1.06 \%)$, our LSC is $2.94 \%$. Applying the LSC to pilot baseline lumbar spine aBMD data for those who have met inclusion for the trial (mean of $1.021 \mathrm{~g} / \mathrm{cm}^{2}$ and $\mathrm{SD}$ of $0.052 \mathrm{~g} / \mathrm{cm}^{2}$ ), we determine from a two-tailed test with power of $80 \%$ and $\alpha=0.05$ that a sample size of 48 will be required for a minimal detectable change of $0.030 \mathrm{~g}$ / $\mathrm{cm}^{2}$. Therefore, we aim to recruit 58 participants into each group to allow for a potential participant dropout rate of up to $20 \%$.

\section{DISCUSSION}

The OPTIMA-Ex trial will be the first trial to investigate the comparative efficacy and safety of a 10-month, supervised, high-intensity, progressive impact loading programme versus a 10-month, supervised, high-intensity progressive resistance training programme on several determinants of bone health of both the upper and lower limbs for healthy young adult women with lower than average bone mass. The comparison of novel exercise programmes specifically designed to target impact-derived versus muscle-derived bone loading has not been fully examined, with minimal direct comparisons in young adult women. ${ }^{19}$ Furthermore, previous work has not examined the comparative effect of 'true' high-intensity loading on bone, on the upper and lower limbs at clinically relevant sites. Moreover, the OPTIMA-Ex trial will use outcome measures that will account for morphological changes to bone at these clinically relevant sites beyond standard bone mineral density measures.

Several limitations warrant justification. First, the outcome assessor is not blind to group allocation after completion of baseline testing and will be the supervising trainer delivering the intervention and evaluating safety. Regrettably, a blinded outcome assessor is beyond the means of the current project; thus, the study design has been adopted out of necessity. We will be implementing a number of strategies to minimise the risk of ascertainment bias, including blinded randomisation, as well as quality assurance procedures that will be conducted on all outcomes measures by a researcher external to the trial. Second, it must be noted that it is difficult to compare overall intensity of the two intervention programmes as they are two intentionally different exercise modalities. However, by designing the exercise programme as high intensity in their respective training domains, the osteogenic nature of the two interventions can be compared. Furthermore, the use of a 'positive' control could be considered a design weakness as we are not comparing the intervention groups to a true non-exercise control group. However, the 'positive' control group was selected to aid in participant compliance, retention and blinding for those assigned to the control group. Further, the 'positive' control acts as an exercise placebo to reduce participant ascertainment bias as those randomly allocated to the control arm of the trial will receive an exercise training programme that has been designed to provoke negligible osteogenic benefit. Finally, our study sample will include sedentary, otherwise healthy young adult women; therefore, our findings may not be applicable to young adult women with pathologically low bone mass or other exclusion characteristics.

In conclusion, the proposed study will assist with the determination of the most effective source of osteogenic mechanical loading (muscle or gravity) and provide grounds for exercise recommendations for the optimisation of peak bone mass in young women. It is intended that the findings of this unique trial examining both the upper and lower limbs will provide further insight into the benefits of high-intensity, bone-targeted exercise programme for osteoporosis prevention.

Acknowledgements The authors would like to acknowledge PUNCH Equipment for special sponsorship pricing on boxing equipment required for the trial.

Contributors Conception and design of the study: CL, BRB, SLW, BKW. Manuscript preparation and editing the final paper for submission: $\mathrm{CL}, \mathrm{ATH}, \mathrm{BRB}, \mathrm{BKW}$. Preparation of information sheets, consent forms and case report forms: $\mathrm{CL}, \mathrm{BRB}$, SLW, BKW. Participant recruitment data collection and participant training: CL. Principle investigator: BKW.

Funding This research is an unfunded $\mathrm{PhD}$ project which received no specific grant from any funding agency in the public, commercial or not-for-profit sectors. The PhD candidate is supported by the Australian Government Research Training Program Scholarship and Griffith University Higher Degree Research student funds.

\section{Competing interests None declared.}

Ethics approval The trial has received ethical approval from the Griffith University Human Research Ethics Committee (GU Ref: 2015/775), and has been prospectively registered with the Australian New Zealand Clinical Trials Registry (\#ACTRN12616001444471).

Provenance and peer review Not commissioned; externally peer reviewed.

Open Access This is an Open Access article distributed in accordance with the Creative Commons Attribution Non Commercial (CC BY-NC 4.0) license, which permits others to distribute, remix, adapt, build upon this work non-commercially, and license their derivative works on different terms, provided the original work is properly cited and the use is non-commercial. See: http://creativecommons.org/ licenses/by-nc/4.0/

(C) Article author(s) (or their employer(s) unless otherwise stated in the text of the article) 2017. All rights reserved. No commercial use is permitted unless otherwise expressly granted. 


\section{REFERENCES}

1. Consensus development conference: prophylaxis and treatment of osteoporosis. Am J Med 1991;90:107-10.

2. Henry MJ, Pasco JA, Nicholson GC, et al. Prevalence of osteoporosis in Australian men and women: Geelong Osteoporosis Study. Med J Aust 2011;195:321-2.

3. Watts JJ, Abimanyi-Ochom J, Sanders KM. Osteoporosis costing all Australians a new burben of disease analysis - 2012 to 2022. Sydney: Osteoporosis Australia, 2013.

4. Saville PD. Osteoporosis: disease or senescence? Lancet 1968;1:535

5. Cummings SR, Black DM, Nevitt MC, et al. Bone density at various sites for prediction of hip fractures. The Study of Osteoporotic Fractures Research Group. Lancet 1993;341:72-5.

6. Abrams SA. Normal acquisition and loss of bone mass. Horm Res 2003;60(Suppl 3):71-6.

7. Rubin CT, Lanyon LE. Regulation of bone mass by mechanical strain magnitude. Calcif Tissue Int 1985;37:411-7.

8. Rubin C, Turner AS, Mallinckrodt C, et al. Mechanical strain, induced noninvasively in the high-frequency domain, is anabolic to cancellous bone, but not cortical bone. Bone 2002;30:445-52.

9. Turner $\mathrm{CH}$, Owan I, Takano Y. Mechanotransduction in bone: role of strain rate. Am J Physiol 1995;269:E438-42.

10. Bolam KA, van Uffelen JG, Taaffe DR. The effect of physical exercise on bone density in middle-aged and older men: a systematic review. Osteoporos Int 2013;24:2749-62.

11. Beck BR. Muscle forces or gravity-what predominates mechanical loading on bone? Introduction. Med Sci Sports Exerc 2009;41:2033-6.

12. Gleeson PB, Protas EJ, LeBlanc AD, et al. Effects of weight lifting on bone mineral density in premenopausal women. J Bone Miner Res 1990;5:153-8.

13. Nickols-Richardson SM, Miller LE, Wootten DF, et al. Concentric and eccentric isokinetic resistance training similarly increases muscular strength, fat-free soft tissue mass, and specific bone mineral measurements in young women. Osteoporos Int 2007;18:789-96.

14. Ballard TL, Specker BL, Binkley TL, et al. Effect of protein supplementation during a 6-month strength and conditioning program on areal and volumetric bone parameters. Bone 2006;38:898-904.

15. Bassey EJ, Ramsdale SJ. Increase in femoral bone density in young women following high-impact exercise. Osteoporos Int 1994;4:72-5.

16. Kato T, Terashima T, Yamashita T, et al. Effect of low-repetition jump training on bone mineral density in young women. J Appl Physiol 2006;100:839-43.

17. Vainionpää A, Korpelainen R, Leppäluoto J, et al. Effects of highimpact exercise on bone mineral density: a randomized controlled trial in premenopausal women. Osteoporos Int 2005;16:191-7.

18. Kelley GA, Kelley KS, Kohrt WM. Exercise and bone mineral density in premenopausal women: a meta-analysis of randomized controlled trials. Int J Endocrinol 2013;2013:741639.

19. Liang MT, Braun W, Bassin SL, et al. Effect of high-impact aerobics and strength training on BMD in young women aged 20-35 years. Int J Sports Med 2011;32:100-8.

20. Martyn-St James M, Carroll S. Effects of different impact exercise modalities on bone mineral density in premenopausal women: a meta-analysis. J Bone Miner Metab 2010;28:251-67.

21. Zhao R, Zhao M, Zhang L. Efficiency of jumping exercise in improving bone mineral density among premenopausal women: a meta-analysis. Sports Med 2014;44:1393-402.

22. Bassey EJ, Rothwell MC, Littlewood JJ, et al. Pre- and postmenopausal women have different bone mineral density responses to the same high-impact exercise. J Bone Miner Res 1998;13:1805-13.

23. Sugiyama T, Yamaguchi A, Kawai S. Effects of skeletal loading on bone mass and compensation mechanism in bone: a new insight into the 'mechanostat' theory. J Bone Miner Metab 2002;20:196-200.

24. Heinonen A, Kannus $P$, Sievänen $H$, et al. Randomised controlled trial of effect of high-impact exercise on selected risk factors for osteoporotic fractures. Lancet 1996;348:1343-7.

25. Vainionpää $A$, Korpelainen $R$, Vihriälä $E$, et al. Intensity of exercise is associated with bone density change in premenopausal women. Osteoporos Int 2006;17:455-63.

26. Witzke KA, Snow CM. Effects of plyometric jump training on bone mass in adolescent girls. Med Sci Sports Exerc 2000;32:1051-7.

27. Lohman T, Going S, Pamenter R, et al. Effects of resistance training on regional and total bone mineral density in premenopausal women: a randomized prospective study. J Bone Miner Res 1995;10:1015-24.

28. Snow-Harter C, Bouxsein ML, Lewis BT, et al. Effects of resistance and endurance exercise on bone mineral status of young women: a randomized exercise intervention trial. J Bone Miner Res 1992;7:761-9.

29. McDermott MT, Christensen RS, Lattimer J. The effects of regionspecific resistance and aerobic exercises on bone mineral density in premenopausal women. Mil Med 2001;166:318-21.

30. Martyn-St James M, Carroll S. Progressive high-intensity resistance training and bone mineral density changes among premenopausal women: evidence of discordant site-specific skeletal effects. Sports Med 2006;36:683-704.

31. Sinaki M, Wahner HW, Bergstralh EJ, et al. Three-year controlled, randomized trial of the effect of dose-specified loading and strengthening exercises on bone mineral density of spine and femur in nonathletic, physically active women. Bone 1996;19:233-44.

32. Kohrt WM, Bloomfield SA, Little KD, et al. American College of Sports Medicine Position Stand: physical activity and bone health. Med Sci Sports Exerc 2004;36:1985-96.

33. Beck BR, Daly RM, Singh MA, et al. Exercise and Sports Science Australia (ESSA) position statement on exercise prescription for the prevention and management of osteoporosis. J Sci Med Sport 2017;20.

34. Granhed $\mathrm{H}$, Jonson $\mathrm{R}$, Hansson $\mathrm{T}$. The loads on the lumbar spine during extreme weight lifting. Spine 1987;12:146-9.

35. Hattin HC, Pierrynowski MR, Ball KA. Effect of load, cadence, and fatigue on tibio-femoral joint force during a half squat. $\mathrm{Med} \mathrm{Sci}$ Sports Exerc 1989;21:613-8.

36. Mosti MP, Carlsen T, Aas E, et al. Maximal strength training improves bone mineral density and neuromuscular performance in young adult women. J Strength Cond Res 2014;28:2935-45.

37. Almstedt HC, Canepa JA, Ramirez DA, et al. Changes in bone mineral density in response to 24 weeks of resistance training in college-age men and women. J Strength Cond Res 2011;25:1098-103

38. Wallace BA, Cumming RG. Systematic review of randomized trials of the effect of exercise on bone mass in pre- and postmenopausal women. Calcif Tissue Int 2000;67:10-18.

39. Cummings SR, Melton LJ. Epidemiology and outcomes of osteoporotic fractures. Lancet 2002;359:1761-7.

40. Cummings SR, Kelsey JL, Nevitt MC, et al. Epidemiology of osteoporosis and osteoporotic fractures. Epidemiol Rev 1985;7:178-208

41. Adami S, Gatti D, Braga V, et al. Site-specific effects of strength training on bone structure and geometry of ultradistal radius in postmenopausal women. J Bone Miner Res 1999;14:120-4.

42. Heinonen A, Sievänen H, Kannus $P$, et al. Effects of unilateral strength training and detraining on bone mineral mass and estimated mechanical characteristics of the upper limb bones in young women. $J$ Bone Miner Res 1996;11:490-501.

43. Wang MY, Salem GJ. The relations among upper-extremity loading characteristics and bone mineral density changes in young women. Bone 2004;34:1053-63.

44. Kanehisa H, Ikegawa S, Fukunaga T. Body composition and crosssectional areas of limb lean tissues in Olympic weight lifters. Scand $J$ Med Sci Sports 1998;8:271-8.

45. Heinonen A, Oja P, Kannus P, et al. Bone mineral density of female athletes in different sports. Bone Miner 1993;23:1-14.

46. Chaabène $\mathrm{H}$, Hachana $\mathrm{Y}$, Franchini $\mathrm{E}$, et al. Physical and physiological profile of elite karate athletes. Sports Med 2012;42:829-43.

47. Morel J, Combe B, Francisco J, et al. Bone mineral density of 704 amateur sportsmen involved in different physical activities. Osteoporos Int 2001;12:152-7.

48. Trutschnigg $B$, Chong $C$, Habermayerova $L$, et al. Female boxers have high bone mineral density despite low body fat mass, high energy expenditure, and a high incidence of oligomenorrhea. Appl Physiol Nutr Metab 2008;33:863-9.

49. Nasri R, Hassen Zrour S, Rebai H, et al. Combat sports practice favors bone mineral density among adolescent male athletes. J Clin Densitom 2015;18:54-9.

50. Clarke B. Normal bone anatomy and physiology. Clin J Am Soc Nephrol 2008;3:S131-9.

51. Chan AW, Tetzlaff JM, Altman DG, et al. SPIRIT 2013 statement: defining standard protocol items for clinical trials. Ann Intern Med 2013;158:200-7

52. Weeks BK, Beck BR. The BPAQ: a bone-specific physical activity assessment instrument. Osteoporos Int 2008;19:1567-77.

53. Rantalainen T, Weeks BK, Nogueira RC, et al. Long bone robustness during growth: A cross-sectional pQCT examination of children and young adults aged 5-29 years. Bone 2016;93:71-8.

54. Weeks BK, Gerrits TA, Horan SA, et al. Muscle size not density predicts variance in muscle strength and neuromuscular 
performance in healthy adult men and women. $J$ Strength Cond Res 2016;30:1577-84.

55. Kim S, Bemben MG, Knehans AW, et al. Effects of an 8-month ashtanga-based yoga intervention on bone metabolism in middleaged premenopausal women: a Randomized Controlled Study. J Sports Sci Med 2015;14:756-68.

56. Southmayd EA, Mallinson RJ, Williams NI, et al. Unique effects of energy versus estrogen deficiency on multiple components of bone strength in exercising women. Osteoporos Int 2017;28:1365-76.

57. National Institutes of Health. Clinical guidelines on the indentification, evaluation, and treatment of overweight and obesity in adults: The evidence report. Bethesda (MD) National Heart, Lung, and Blood Institute; 1998 Sep, Report No.: 98-4083. https://www.nhlbi.nih.gov/ files/docs/guidelines/prctgd_c.pdf

58. Little A, Harding A, Weeks BK, et al. Validity and reliability of a simple lower extremity strength test. Gold Coast, QLD, Australia: Gold Coast Health \& Medical Research Conference 2014, 2014.

59. Harding AT, Weeks BK, Horan SA, et al. Validity and test-retest reliability of a novel simple back extensor muscle strength test. SAGE Open Med 2017;5:205031211668884.

60. Linthorne NP. Analysis of standing vertical jumps using a force platform. Am J Phys 2001;69:1198-204.

61. Weeks BK, Beck BR. The BPAQ: a bone-specific physical activity assessment instrument. Osteoporos Int 2008;19:1567-77.
62. Weeks BK, Hirsch RD, Moran D, et al. A useful tool for analysing the effects of bone-specific physical activity. Salud Ciencia 2011;18:538-42.

63. Beck BR, Weeks BK, Norling TL. A novel Australian calciumspecific diet questionnaire: Validity and reliability. Osteoporis Int 2011;22:S626-7.

64. Richardson JR, Peacock SJ, Hawthorne G, et al. Construction of the descriptive system for the Assessment of Quality of Life AQoL-6D utility instrument. Health Qual Life Outcomes 2012;10:38.

65. Hawthorne G, Richardson J, Osborne R. The Assessment of Quality of Life (AQoL) instrument: a psychometric measure of health-related quality of life. Qual Life Res 1999;8:209-24.

66. Hawthorne G, Richardson J, Day NA. A comparison of the Assessment of Quality of Life (AQoL) with four other generic utility instruments. Ann Med 2001;33:358-70.

67. Hawthorne G, Osborne R. Population norms and meaningful differences for the Assessment of Quality of Life (AQoL) measure. Aust N Z J Public Health 2005;29:136-42.

68. Mullen SP, Olson EA, Phillips SM, et al. Measuring enjoyment of physical activity in older adults: invariance of the physical activity enjoyment scale (paces) across groups and time. Int J Behav Nutr Phys Act 2011;8:103.

69. Baim S, Wilson CR, Lewiecki EM, et al. Precision assessment and radiation safety for dual-energy $\mathrm{X}$-ray absorptiometry. J Clin Densitom 2005;8:371-8. 